\title{
Evaluation of spermicidal potential of labdane diterpenes from Andrographis paniculata
}

\author{
Shrabanti KUMAR, Subhendu NASKAR, Abhijit HAZRA, Sohini SARKAR, \\ Nirup B. MONDAL and Sukdeb BANERJEE * \\ Department of Chemistry, Indian Institute of Chemical Biology, Council of Scientific and \\ Industrial Research, 4 Raja SC Mullick Road, Jadavpur, Kolkata- 700 032, India. \\ *Corresponding author, E-mail: sukdeb@iicb.res.in
}

\begin{abstract}
Andrographolide, 14-deoxyandrographolide, andrograpanin, andrographiside, deoxyandrographiside, and neoandrographolide were isolated from the leaves of A. paniculata and evaluated for their spermimmobilizing activity. The mode of spermicidal action was assessed by (a) supravital and double fluoroprobe staining of sperm, (b) hypo-osmotic swelling test, and (c) transmission electron microscopy. Contraceptive efficacy was evaluated by intrauterine application in rat followed by mating, and subsequent assessment of the pregnancy outcome. 14-Deoxyandrographolide with MEC of $200 \mu \mathrm{g} / \mathrm{ml}$ was found to be the most active among the six compounds. It is comparable in activity to nonoxynol (N-9) and exerts its effect in a dose dependent manner. Intra-vaginal administration of 10\% 14-deoxyandrographolide did not cause any significant vaginal irritation in rat. The findings strongly suggest that 14-deoxyandrographolide may be used as a nondetergent type spermicidal agent.
\end{abstract}

(C) 2009 International Formulae Group. All rights reserved.

Key words: sperm-immobilizing activity; andrographolide; 14-deoxyandrographolide.

\section{INTRODUCTION}

Population explosion is a challenge to the quality of life particularly in the underdeveloped and developing countries. A suitable contraceptive device is primarily required to combat this grave situation. Though detergent type vaginal contraceptive products based on nonoxynol-9 (N-9) have been available for quite a long time, clinical studies have revealed that frequent use of $\mathrm{N}-9$ alters normal vaginal flora and leads to increased risks of spreading sexually transmitted diseases (Zaneveld, 1984; Trussell, 1994; Roddy et al., 1998; Staford et al., 1998). This invites attention for a safer alternative spermicidal agent for which natural products appear to be particularly attractive.

$$
\text { Andrographis paniculata Nees }
$$

(Acanthaceae), a small herb abundantly available in South East Asia particularly in Indian sub-continent and China, is one of the commercially exploited traditional medicinal plants used for various ailments. In the Ayurvedic system of medicine, the plant is used extensively for the treatment of diarrhoea, inflammation, fever, cold, and laryngitis (Chadha, 1985). It is reported that extracts of this plant and its constituents display a wide range of biological activities like hepatoprotective (Handa and Sharma, 1990), immuno-stimulant (Puri et al., 1993), anti-inflammatory (Deng, 1990), anti-malarial (Dua et al., 2004), and anti HIV (Calabrese et al., 2000; Reddy et al., 2005). Methanol extract of this plant exhibited potent cell differentiation inducing activity in myeloid leukemia (M1) cells (Matsuda et al., 1994). 
The major chemical constituents identified from this plant are labdane type diterpenoids and their glucosides like andrographolide, neoandregrapholide etc. Although several diterpenes, e.g. triptolide and kauranoic acid (Lue et al., 1998; Ambrosio et al., 2006), have been reported to exert spermicidal activity, the diterpenoids and their glycosides of $A$. paniculata do not appear to have been screened for this purpose.

As a part of our continuous search for bioactive secondary plant metabolites (Mandal et al., 2006; Pramanick et al., 2006; Dutta et al., 2007), we carried out investigation on $A$. paniculata leaves leading to the isolation of three labdane diterpene lactones along with their glucosides and decided to screen their spermicidal activity using nonoxynol-9 (N-9) as positive control. The results revealed that the spermicidal activity of 14-deoxyandrographolide is comparable to that of N-9 (Shah et al., 2005) and is exerted in a dose dependent manner. The compound may therefore be explored for developing a safer spermicide cum vaginal contraceptive.

\section{MATERIALS AND METHODS Plant material}

The leaves of $A$. paniculata were collected from the suburbs of Kolkata in March 2007 and identified at the Indian Botanical Garden, Howrah, India. A voucher specimen (No. 227) has been deposited in the Department of Chemistry, Indian Institute of Chemical Biology.

\section{Extraction and isolation}

The air-dried powdered leaves of $A$. paniculata $(1 \mathrm{~kg})$ were defatted with petroleum ether $\left(60-80{ }^{\circ} \mathrm{C}\right)$ and successively extracted with $\mathrm{CHCl}_{3}(3 \times 5 \mathrm{~L})$ and $\mathrm{MeOH}$ $(3 \times 5$ L) at ambient temperature. The combined $\mathrm{MeOH}$ extract was concentrated under reduced pressure and partitioned between $n$ - $\mathrm{BuOH}$ and $\mathrm{H}_{2} \mathrm{O}$. The organic layer was washed with water, dried, and evaporated under reduced pressure to yield a dark brown residue (18 g). Chromatography of the residue yielded andrographolide $(\mathbf{1}, 6.8 \mathrm{~g}), \quad 14-$ deoxyandrographolide (2, $120 \quad \mathrm{mg})$, andrograpanin (3,18 $\mathrm{mg})$, andrographiside (4, $12 \mathrm{mg})$, deoxyandrographiside $(\mathbf{5}, 24 \mathrm{mg})$, and neoandrographolide (6, $210 \mathrm{mg}$ ) (Figure1). The compounds were characterized by the application of various spectroscopic methods and comparison of spectral data reported in literature (Matsuda et al., 1994; Pramanick et al., 2006).

\section{Chemicals}

Analytical grade chemicals and solvents were procured from Merck India Ltd., eosin-nigrosin from Sisco Research Laboratory (India), and LIVE/DEAD Sperm Viability Kit from Molecular Probes Inc (USA).

\section{Animals}

Adult Sprague-Dawley rats (190-220 g) were acquired from our institute's animal facility and maintained under controlled light and temperature (complying with standard husbandry conditions) with food and water ad libitum. Sperm were collected from adult male rats of proven fertility (Adult male rats chosen for sperm collection was subjected for mating with adult female rats in their proestrous stage. Successful mating was confirmed by checking the vaginal lavage). All experiments were performed following the guidelines formulated by the animal ethics
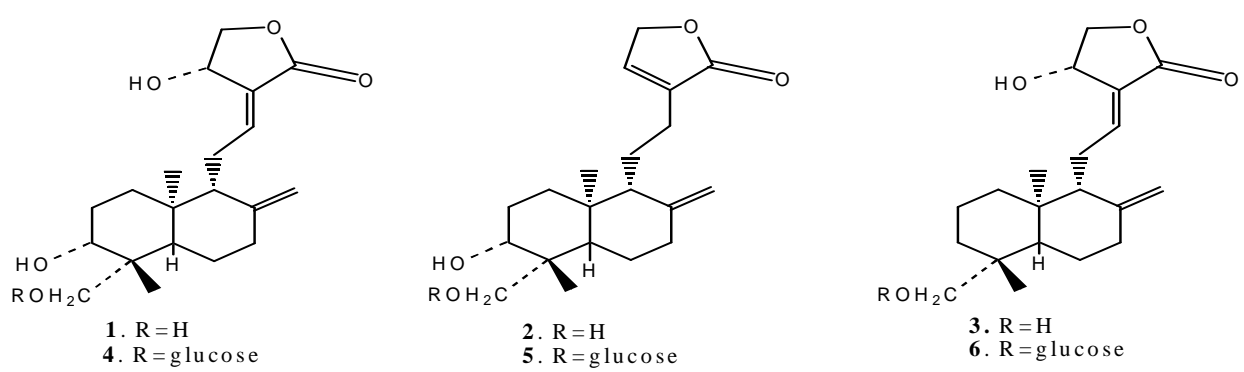

Figure 1: Structures of diterpene lactones andrographolide (1), 14-deoxyandrographolide(2), andrograpanine(3), andrographiside(4), deoxyandrographiside(5), and neoandrographolide(6). 
committee of the Institute. Sperm suspension having highly motile sperm with avarage motility $85-90 \%$ were taken.

\section{Isolation of caudal rat sperm exudates}

Sperm were collected from adult male rats, which have been proven to be fertile, after sexual abstinence for at least 3 days. The animals were anesthetized with ketamine (100 $\mathrm{mg} / \mathrm{kg}$ body weight) followed by cervical dislocation, and sperm were obtained from cauda epididymides. The epididymides were dissected out of the testis, and the caudal region was identified and pricked carefully with the aid of a sterilized needle (18G), avoiding any blood vessel rupture (Kumar et al., 2007). The exudates were collected and suspended in pre-equilibrated Biggers, Whitten and Whittingham (BWW) medium [94 mM NaCl, $4.7 \mathrm{mM} \mathrm{KCl}, 1.7 \mathrm{mM} \mathrm{CaCl}_{2}$, $1.2 \mathrm{mM} \mathrm{KH} \mathrm{PO}_{4}, 1.2 \mathrm{mM} \mathrm{MgSO}{ }_{4} 7 \mathrm{H}_{2} \mathrm{O}, 25$ $\mathrm{mM} \mathrm{NaHCO}, 0.5 \mathrm{mM}$ sodium pyruvate, 19 $\mathrm{mM}$ sodium lactate, $5 \mathrm{mM}$ glucose, $0.4 \%$ BSA, $0.1 \%$ antibiotic (penicillin/streptomycin) solution, $\mathrm{pH}$ 7.2]. The sperm were allowed to swim up in the medium for $30 \mathrm{~min}$ under oil cover in a $\mathrm{CO}_{2}$ incubator at $37{ }^{\circ} \mathrm{C}$. Cells were counted using a Makler chamber. Sperm suspension having highly motile sperm with sperm count $20-25 \times 10^{6} / \mathrm{ml}$ was used for the studies.

\section{Evaluation of spermicidal activity}

The spermicidal activity was determined by following a modified version of the Sander-Cramer test (Khillare and Shrivastav, 2003; Kumar et al., 2007). Stock solutions $(0.5 \% \mathrm{w} / \mathrm{v})$ of the compounds (1-6) were prepared in DMSO and subsequently diluted in BWW medium to yield the desired concentration. Nonoxynol-9 (N-9) diluted in BWW $(0.2 \% \mathrm{w} / \mathrm{v})$. A corresponding volume $50 \mu \mathrm{l}$ of DMSO $(5 \%)$ was added to the control tube. The test compounds were used at concentrations of $0.25,0.5,1.0$ and 2.0 $\mathrm{mg} / \mathrm{ml}$. The test compound solutions $(100 \mu \mathrm{l}$ each) were mixed with an aliquot of $20 \mu \mathrm{l}$ of sperm suspension $\left(25 \times 10^{6} / \mathrm{ml}\right)$ and observed under a phase contrast microscope (100X) for 20 seconds. The numbers of motile sperm were counted using a Makler's chamber. The sperm that lost complete motility within 20 seconds after treatment with the compounds 1-6 were subsequently tested for motility revival. A $250 \mu \mathrm{l}$ aliquot of Baker's buffer (glucose $3 \%, \mathrm{Na}_{2} \mathrm{HPO}_{4} .2 \mathrm{H}_{2} \mathrm{O} 0.31 \%, \mathrm{NaCl}$ $0.2 \%, \mathrm{KH}_{2} \mathrm{PO}_{4} 0.01 \%$ ) was added to the test solutions when total immobilization was observed. The mixture was incubated at $37^{\circ} \mathrm{C}$ for $60 \mathrm{~min}$ and observed again for any motility. Complete immobilization (100\%) within 20 seconds with no revival of motility in the buffer after $60 \mathrm{~min}$ incubation at $37^{\circ} \mathrm{C}$ was considered to be the minimum effective concentration (MEC).

\section{Hypo-osmotic swelling test}

The hypo-osmotic swelling (HOS) test was performed (Jayendran et al., 1984) for evaluation of functional integrity of sperm membrane after treatment with each of 1-6. The sperm suspensions were treated with each of 1-6 at different concentrations $(0.25,0.5$, 1.0 and $2.0 \mathrm{mg} / \mathrm{ml}$ ) along with control, and were then exposed to HOS solution $(75 \mathrm{mM}$ fructose and $20 \mathrm{mM}$ sodium citrate) for 30 min at $37{ }^{\circ} \mathrm{C}$. The number of sperm showing tail coiling or characteristic swelling was counted under a phase contrast microscope $(400 \mathrm{X})$ and 100 sperm were considered in each counting.

\section{Sperm viability test}

Assessment of sperm viability was accomplished by using eosin-nigrosin staining technique. Eosin stained sperm were counted as dead whereas unstained ones were counted as live (Maikuri et al., 2003). For counting, 200 sperm were taken under a phase contrast microscope (400X).

\section{Sperm viability test by fluorescent staining}

Viability of sperm was also examined by a double fluoroprobe staining method (Sharma et al., 1996). An aliquot (1 ml) of sperm treated with 14-deoxyandrographolide $(200 \mu \mathrm{g} / \mathrm{mL})$ was mixed with $5 \mu \mathrm{L}$ of 50 -fold diluted stock solution of a fluorescent dye, SYBR14 (Invitrogen, USA) and $5 \mu \mathrm{L}$ of propidium iodide (PI) from the stock, and the mixture was incubated for 10 minutes. A drop of the mixture was placed on a slide and viewed under fluorescence microscope (Olympus BX51, Tokyo, Japan).

\section{Ultra structural study}

An aliquot of each sperm suspension control and treated with 14deoxyandrographolide $(200 \mu \mathrm{g} / \mathrm{ml})$ were fixed 
with $2 \%$ glutaraldehyde in phosphate buffer at $4{ }^{\circ} \mathrm{C}$ for 4 hour. After three successive washings in buffer, post fixation was done using $1 \%$ osmium tetra oxide for observation under transmission electron microscope (TEM) (Pakrashi et al., 1991).

\section{Intra uterine administration of 14- deoxyandrographolide in rats}

In vivo spermicidal potential of 14deoxyandrographolide was evaluated in experimental rat model as described (Weitlauf, 1994). Regularly cycling adult Sprague Dawley rats $(n=6)$ were subjected to light ether anesthesia on the day of proestrus phase of their cycle. The two uterine horns were gently pulled out through a small mid ventral abdominal incision. In one uterine horn $50 \mu \mathrm{l}$ sterile solution of 14deoxyandrographolide $(2 \mathrm{mg} / \mathrm{ml})$ was administered through a tuberculin syringe fitted with a 24-G needle that penetrated through the cervical end (treated horn), while $50 \mu \mathrm{l}$ of vehicle was introduced in the contralateral uterine horn (control horn). The incision was closed by sutures. The animals were maintained under proper postoperative care, kept in individual cages, and each of the compound treated females was exposed to adult male rats with proven fertility in a 1:1 ratio. Vaginal lavage was examined under microscope to check the presence of sperm on the following day and was designated as day 1 of pregnancy. The rats were autopsied on day 8 of pregnancy. The uterine horns were dissected out to examine the presence of implantation sites.

\section{Vaginal irritation test}

For the vaginal irritation study (D'Cruz and Uckun, 2001) 24 adult Sprague Dawley rats were divided into three groups. One group was administered with $0.1 \mathrm{ml}$ of $\mathrm{K}-\mathrm{Y}$ jelly alone (placebo) and the other two groups were administered with $0.1 \mathrm{ml}$ of $\mathrm{K}-\mathrm{Y}$ jelly based $2 \%$ and $10 \%$ 14-deoxyandrographolide for 14 consecutive days. On day 15 , animals were sacrificed by excess anaesthesia using ketamine (100 mg/kg body weight). The vagina tissues were split opened and fixed in $10 \%$ formalin overnight. The fixed tissues were then embedded in paraffin, sectioned at a thickness of $5 \mu \mathrm{m}$, stained with Harris hematoxylin and eosin, and examined under light microscope (200X).

\section{Statistical analysis}

The data are expressed as mean \pm SEM and analyzed by student's $t$-test. $\mathrm{P}<0.5$ was considered as the criterion for statistical significance.

\section{RESULTS}

Sperm-immobilizing potential

Sander-Cramer test exhibited that among the six compounds 14deoxyandrographolide was the most potent, exerting its effect in a dose dependent manner (Figure 1 and Figure 2; Table-1). The sperm immobilization activity was quite comparable with that of nonoxynol-9. The minimum effective concentration (MEC) for $100 \%$ immobilization of sperm was found to be 200 $\mu \mathrm{g} / \mathrm{ml}$ (Table 1).

\section{Sperm membrane integrity}

14-Deoxyandrographolide-treated sperm exhibited loss of their response to hypoosmotic medium in a dose dependent manner while more than $98 \%$ of the control spermatozoa responded to hypoosmotic solution by swelling/curling of their tail.

\section{Sperm viability test}

Microscopic examination of the sperm treated with 14-deoxyandrographolide at MEC revealed that the heads of almost all the spermatozoa took eosin stain in each batch of experiment while in the control sets most of the spermatozoa remained unstained.

\section{Sperm viability test by fluorescent staining}

Fluorescence microscopic examination of sperm treated with 14-deoxyandrographolide confirmed the loss of their viability upon fluorescent double staining with SYBR14 and propidium iodide, the majority of the sperm $>90 \%$ from the control set appeared green, while those from the 14deoxyandrographolide treated group emitted red fluorescence (Figures 3A, 3B).

\section{Ultra structural studies}

Ultra structural examination revealed loss of membrane integrity in 14deoxyandrographolide treated sperm while structurally intact plasma membrane was 


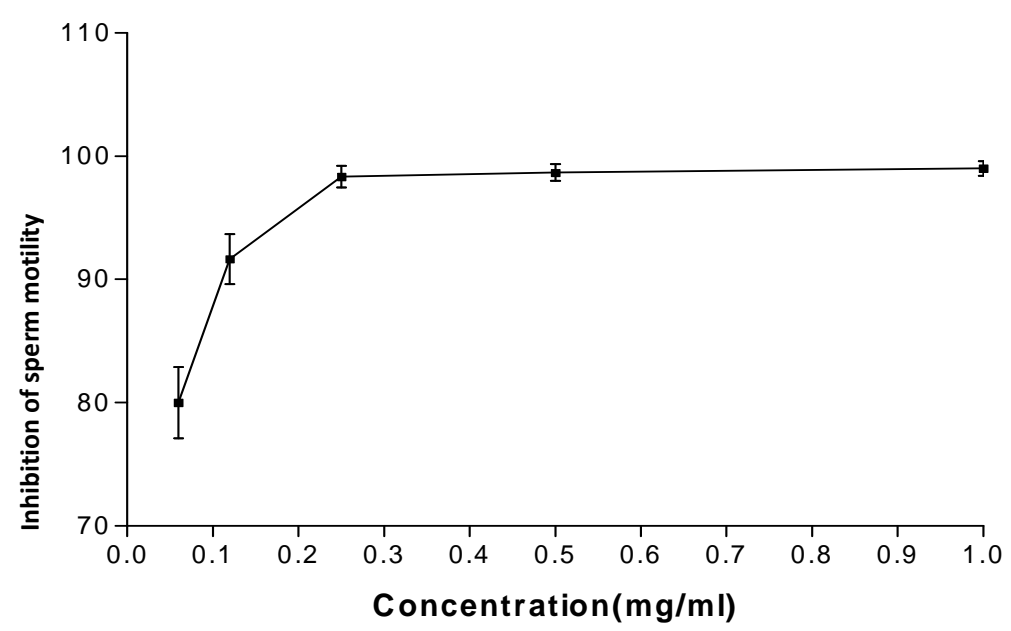

Figure 2: Concentration dependent inhibition of sperm motility.

Table 1: Minimum effective concentration (MEC) of compound 1-6 and N-9 required to kill 1 million sperm in 20 seconds.

\begin{tabular}{lccc}
\hline Compound & $\begin{array}{c}\text { Sperm count } \\
(\mathbf{m i l l i o n} / \mathbf{m l})\end{array}$ & $\begin{array}{c}\text { Amount of sperm suspension taken } \\
(\boldsymbol{\mu l})\end{array}$ & $\begin{array}{c}\text { MEC } \\
(\mathbf{m g} / \mathbf{m l})\end{array}$ \\
\hline 1 & 50 & 20.0 & $1.1 \pm 0.058$ \\
2 & 48 & 21 & $0.21 \pm 0.009$ \\
3 & 70 & 14.2 & $2.07 \pm 0.037$ \\
4 & 60 & 16.7 & $0.96 \pm 0.012$ \\
5 & 55 & 18.1 & $0.24 \pm 0.006$ \\
6 & 72 & 13.8 & $2.2 \pm 0.058$ \\
$\mathrm{~N}-9$ & 54 & 19.2 & $0.20 \pm 0.003$ \\
\hline
\end{tabular}

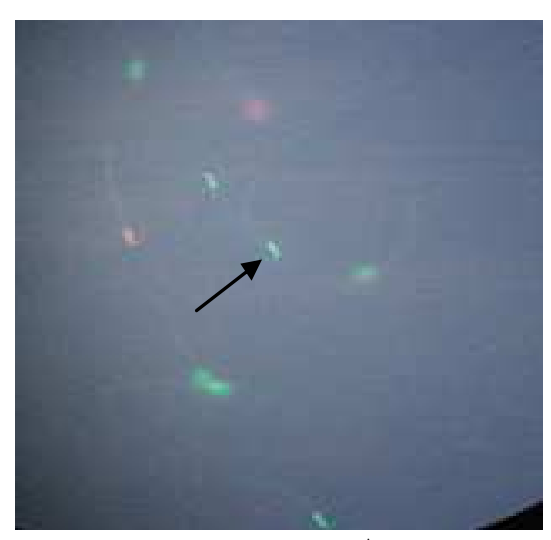

A

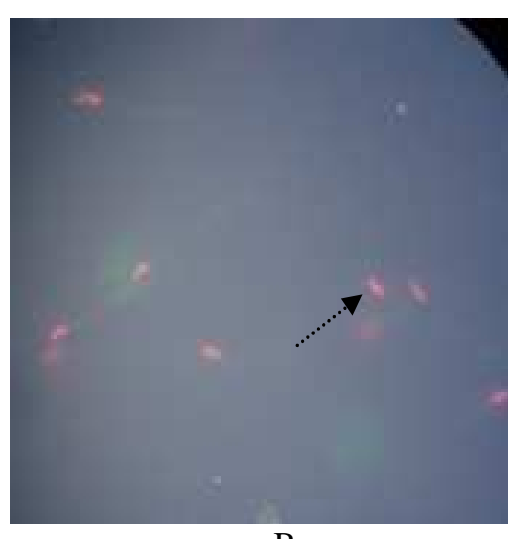

B

Figure 3: Photomicrograph of SYBR14/PI staining. (A) Control sperm subjected to double fluoroprobe staining; (B) 14-deoxoandrographolide treated sperm. Sperm head appeared green due to binding of SYBR14 with the sperm chromatin and treated sperm appeared red due to uptake of PI. 


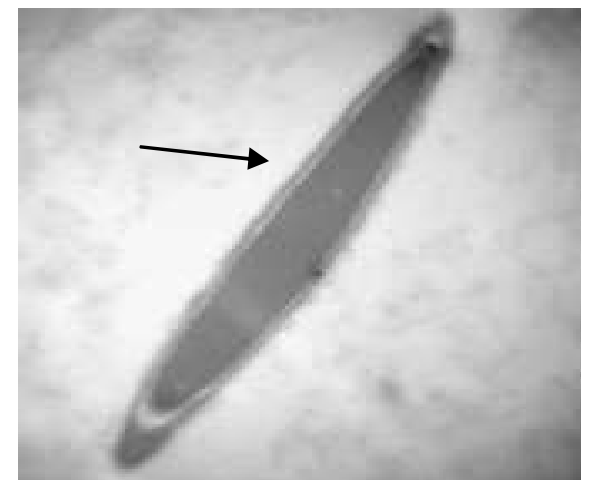

A

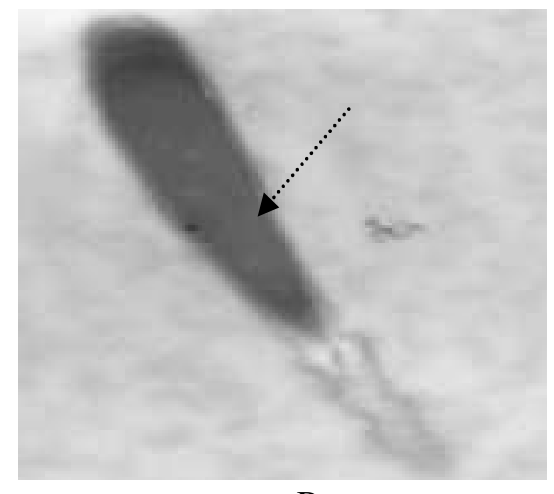

B

Figure 4: Transmission electron micrograph of sperm sample incubated with or without 14deoxyandrographolide at MEC. (A) Control spermatozoa showed proper acrosomal membrane while (B) 14deoxyandrographolide treated sperm exhibit dissolution of the acrosomal cap. Sperm chromatin with proper acrosomal membrane and treated spermchromatin with structurally dissolved membrane.

observed for control spermatozoa (Figures 4A, 4B).

\section{Contraceptive efficacy in rats}

All rats exhibited vaginal presence of sperm which confirmed mating. On day 8 of pregnancy the animals were sacrificed. The saline-treated control uterine horn showed the presence of beadlike embryonic swellings oriented along the length of the uterine horn. By contrast, no viable implantation site (Table-2) was recorded in the 14deoxyandrographolide treated uterine horn.

\section{Vaginal irritation test}

Intra-vaginal administration of $2 \% 14$ deoxyandrographolide (100 times more than MEC) for 14 consecutive days revealed no sign of local toxicity in any of the treated rats, but the animals receiving $10 \%$ of 14 deoxyandrographolide for 14 days showed little vaginal tissue inflammation along with leukocyte infiltration when observed under light microscope (Figures 5A, 5B, 5C).

\section{DISCUSSION}

The investigation was conducted on six different compounds (1-6) isolated from $A$. paniculata to test their sperm immobilizing and contraceptive efficacy in rats. Among the six compounds 14-deoxyandrographolide (2) was found to be the most potent (Table-1) for immobilization of sperm. It was comparable in efficacy with nonoxynol-9 (MEC 250 $\mu \mathrm{g} / \mathrm{ml}$ ) (Shah et al., 2005) and exerts its effect in a dose dependent manner (Figure 2). The MEC of 14-deoxyandrographolide was found to be $200 \mu \mathrm{g} / \mathrm{ml}$, and the effect was adjudged spermicidal because no revival of sperm motility was observed after incubation of $\mathbf{2}$ treated sperm in Baker's buffer. It was also observed that 14-deoxyandrographolide is 5 fold more potent than andrographolide for immobilization of sperm in vitro (Table 1). Considering the potentiality of 14deoxyandrographolide (2) for immobilization of sperm a series of in vitro and in vivo experiments were carried out to establish its spermicidal efficacy.

The plasma membrane plays a vital role in the process of sperm migration and fertilization, and is the target site for a number of spermicidal agents. Generally these agents bring some changes in the structure and function of the plasma membrane (Sharma et al., 1996). The functional changes of sperm membrane caused by treatment with 14deoxyandrographolide were observed through Hypo-osmotic swelling (HOS) test (Jayendran et al., 1984) and eosin-nigrosin staining, which indicated that the spermicidal efficacy was exerted through damage caused in the sperm membrane. Intact cell membrane allows free flow of fluids into the cell to attain osmotic equilibrium when exposed to a hypoosmotic environment; consequently the volume of the cell increases and the plasma membrane bulges. Since the plasma membrane around the sperm tail fiber is more loosely attached than that around other parts, 
Table 2: Implantation site count (on day 8 pc) in the control and compound 2 treated (50 $\mu 1$ of $2 \mathrm{mg} / \mathrm{ml}$ ) uterine horn in rat.

\begin{tabular}{|c|c|c|c|c|}
\hline Animal & No. of implantation site & & Mean \pm SEM & \\
\hline & $\mathrm{CH}$ & TH & ${ }^{*} \mathrm{CH}$ & ${ }^{*} \mathrm{TH}$ \\
\hline 1 & 6 & 0 & \multirow{7}{*}{$6.33 \pm 0.76$} & \multirow{7}{*}{$0.33 \pm 0.22^{* * *}$} \\
\hline 2 & 8 & 1 & & \\
\hline & & & & \\
\hline 3 & 3 & 0 & & \\
\hline 4 & 6 & 0 & & \\
\hline 5 & 8 & 1 & & \\
\hline 6 & 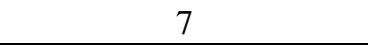 & 0 & & \\
\hline
\end{tabular}

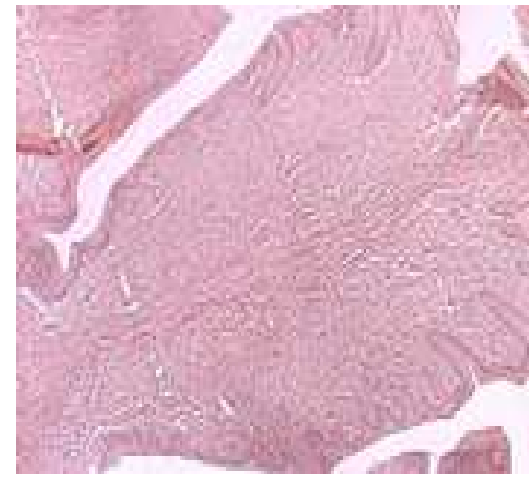

A

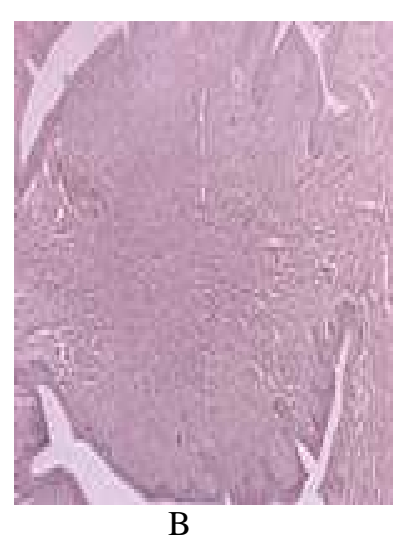

B

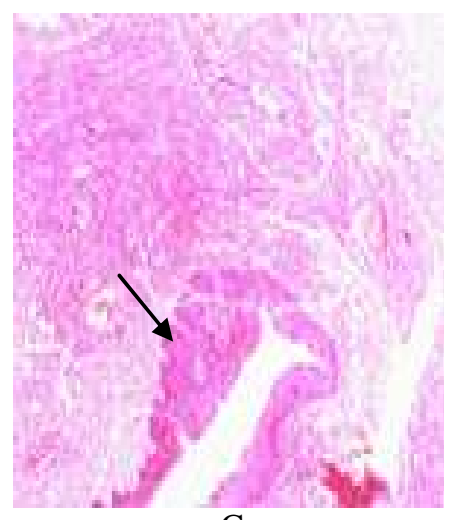

$\mathrm{C}$

Figure 5: Light microscopy images (Original magnification 200X) of 14-deoxyandrographolide treated rat vaginal sections. Representative hematoxilin- and eosin-stained, paraffin embedded sections of the rat vagina treated intra vaginally with K-Y jelly - based (A) 0\% (placebo), (B) 2\%, (C) 10\% 14-deoxyandrographolide. The intact nature of the vaginal epithelial tissue and lack of significant leukocyte infiltration (mark of inflammation) is evident in the treated tissues (Band C). Tissue inflamation along with leukocyte infiltration.

sperm tail is very much susceptible to hypoosmotic exposure and responds by coiling. This characteristic feature was observed in $>98 \%$ of the sperm in the control set, while the sperm treated with 2 at MEC $(200 \mu \mathrm{g} / \mathrm{ml})$ showed only <2\% coiling in morphology. Morphological distortion was also evident from the differential reactions of the normal sperm and treated sperm to eosin-nigrosin and SYBR14/PI fluorescent staining. Generally live sperm with functionally intact membrane offers selective permeability and restricts entry of stains like eosin Y and PI. More than $96 \%$ of control sperm showed no stain of eosin and appeared entirely green due to SYBR-14 only, when subjected to dual fluorescent staining. In contrast, almost all sperm treated with 2 stained positive with eosin and emitted red fluorescence due to entry of PI (Figure 3). These observations strongly suggest that treatment with $\mathbf{2}$ causes loss of functional integrity of the sperm membrane. The transmission electron micrographic observation of 14deoxyandrographolide $(200 \mu \mathrm{g} / \mathrm{ml})$ treated sperm also supported the occurrence of damage to membrane structure. Figure 4 clearly reveals that the membrane architecture of the sperm was lost with dissolution of the outer acrosomal cap, which might have caused loss of functional integrity.

The advantage of the bi-cornuate uterus of rat was utilized for evaluation of spermicidal potential of $\mathbf{2}$ in vivo (Weitlauf, 1994). Thus, $50 \mu \mathrm{l}$ of $2(2 \mathrm{mg} / \mathrm{ml})$ was administered directly into one uterine horn leaving the other one as control and both the horns were exposed to identical systemic 
milieu (Kumar et al., 2007). Results (Table 2) indicated that administration of $\mathbf{2}$ prevented establishment of pregnancy (as observed Day $8 \mathrm{pc})$. On the other hand, implantation occurred unhindered in the control horn. This observation indicated that $\mathbf{2}$ retained its spermicidal potential even in the uterine milieu.

Vaginal irritation test is a measure of local toxicity and can be evaluated by histological examination of respective tissue sections. Intra-vaginal administration of $2 \%$ 14-deoxyandrographolide (100 times more than MEC) for 14 consecutive days showed no sign of local toxicity in any experimental rats. However, little damage of vaginal tissue was observed (Figure 5) after applying $10 \%$ of 2 (500 times more than MEC) for the same period compared to the control (KY jelly based placebo).

Although andrographolide (1) is the major one among the constituents of Andrographis paniculata but 14-deoxyandrographolide (2) is not a very minor one. Synthetically also $\mathbf{2}$ can be prepared from andrographolide (Balmain and Connolly 1973). To the best of our knowledge this is the first report of spermicidal potential of 14deoxyandrographolide (2). The foregoing findings, easy availability of the plant material, and the simplicity of isolation procedure ensure that the future of 14deoxyandrographolide as a spermimmobilizing agent is highly promising. However, further investigation is required to understand the exact mechanism of action by which 14-deoxyandrographolide arrests sperm motility.

\section{ACKNOWLEDGEMENTS}

This work received financial assistance from the Council of Scientific and Industrial Research (Net Work Project-0009), Government of India. S.K., S.N, A.H are recipients of Senior Research Fellowship from CSIR. Authors wish to thank Mr Rajendra Mahato, Mr S.N. Dey and Dr Sibabrata Mukhopadhyay of this institute for collection of plant material, electron microscopy and helpful suggestions.

\section{REFERENCES}

Ambrosio SR, Tirapelli CR, da Costa FB, de Oliveira AM. 2006. Kaurane and pimarane-type diterpenes fron the Viguiera species inhibit vascular smooth muscle contractility. Life Science, 79: 925-933.

Balmain A, Connolly JD. 1973. Minor diterpenoid constituents of Andrographis paniculata Nees. J. Chem. Soc. Perkin I, 1247-1251.

Calabrese C, Berman SH, Babish JG, Ma X, Shinto L, Dorr M, Wells K, Wenner CA, Standish LJ. 2000. A Phase-I trial of andrographolide in HIV positive patients and normal volunteers. Phytother. Res., 14: 333-338.

Chadha YR. 1985. The Wealth of India, Raw Materials (Vol.1A Revised). Publication and Information Directorate (CSIR): New Delhi; 264-266.

D’Cruz OJ, Uckun FM. 2001. Intravaginal toxicity studies of a gel-microemulsion formulation of spermicidal vanadocenes in rabbits. Toxicol. Applied Pharmacol., 170: 104-112.

Deng WL. 1990. Anti-inflammatory agents from traditional Chinese drugs. Drugs Future, 15: 809-816.

Dua VK, Ojha VP, Roy R, Joshi BC, Valecha N, Devi CU, Bhatnagar MC, Sharma VP, Subbarao SK. 2004. Anti-malarial activity of some xanthones isolated from the roots of Andrographis paniculata. J. Ethnopharmocol., 95: 247- 251.

Dutta A, Ghoshal A, Mandal D, Mondal NB, Banerjee S, Sahu NP, Mandal C. 2007. Racemoside A, an anti-leishmanial watersoluble, natural steroidal saponin, induces programmed cell death in Leishmania donovani. Antimicrob Agents Chemother, 56: 1196-1204.

Handa SS, Sharma A. 1990. Hepatoprotective activity of andrographolide against galactosamine and paracetamol intoxication in rats. Indian J. Med. Res., 92: 284- 292.

Jayendran RS, Van der Ven HH, Perag-Pelaez M, Crab BG, Zaneveld LJD. 1984. Development of an assay to assess the functional integrity of the human sperm membrane and its relationship to other semen characteristics, J. Reprod. Fertil., 70: 219- 228.

Khillare B, Shrivastav G. 2003. Spermicidal activity of Azadirachta indica (neem) leaf extract. Contraception, 68: 225- 229.

Kumar S, Biswas S, Mandal D, Roy HN, Chakraborty S, Kabir SN, Banerjee S, Mondal NB. 2007. Chenopodium album seed extract: a potent sperm-immobilizing 
agent both in vitro and in vivo. Contraception, 75: 71-78.

Kumar S, Chatterjee R, Dolai S, Adak S, Kabir SN, Banerjee S, Mondal NB. 2008. Chenopodium album seed extractinduced sperm cell death: exploration of a plausible pathway. Contraception, 77: 456- 462.

Lue Y, Hikim APS, Wang C, Baravarian S, Reutrakul V, Sangsawan R, Chaichana S, Swerdloff RS. 1998. Triptolide: A potential male contraceptive. $J$. Andrology, 19: 479-486.

Maikuri JP, Dwivedi AK, Dhar JD, Setty BS, Gupta G. 2003. Mechanism of action of some acrylophenones, quinolines and dithiocarbamates as potent, nondetergent spermicidal agent. Contraception, 67: 403- 408.

Mandal D, Panda N, Kumar S, Banerjee S, Mandal NB, Sahu NP. 2006. A triterpenoid saponin possessing antileishmanial activity from the leaves of Careya arborea. Phytochemistry, 67: 183-190.

Matsuda T, Kuroyanagi M, Sugiyama S, Umehara K, Ueno A, Nishi K. 1994. Cell differentiation-inducing diterpenes from Andrographis paniculata Nees. Chem. Pharm. Bull., 42: 1216-1225.

Pakrashi A, Roy H, Pal BC, Mahato SB. 1991. Sperm immobilizing effect of triterpene saponins from Acacia auriculiformis. Contraception, 43: 475- 483.

Pramanick S, Banerjee S, Achari B, Das B, Sen, AK Sr, Mukhopadhyay S, Neuman A, Prange T. 2006. Andropanolide and Isoandrographolide, minor diterpenoids from Andrographis paniculata: Structure and X-ray crystallographic analysis. $J$. Nat. Prod., 69: 403-405.

Pramanick S, Banerjee S, Achari B, Mukhopadhyay S. 2007. Phytochemicals from the genus andrographis. In Recent Progress in Medicinal Plant; Phytomedicines, Govil JN, Singh VK, Bhardwaj R (eds). Studium Press LLC Houston: USA; 16: 339-387.

Puri A, Saxena A, Saxena RP, Saxena KC, Srivastava V, Tandon JS. 1993. Immunostimulant agents from Andrographis paniculata. J. Nat. Prod., 56: 995- 999.

Reddy VLN, Reddy SM, Ravikanth V, Krishnaiah P, Goud TV, Rao TP, Ram
TS, Gonnade RG, Bhadbhade M, Venkateswarlu Y. 2005. A new bisandrographolide ether from Andrographis paniculata Nees and evaluation of antiHIV activity. Nat. Prod. Res., 19: 223230.

Roddy RE, Zeking L, Ryan KA, Tamoufe U, Weir SS, Wong EL. 1998. A controlled trial of nonoxynol-9 film to reduce male to female transmission of sexually transmitted diseases. N. Engl. J. Med., 339: 504-510.

Shah V, Doncel GF, Seyoum T, Eaton KM, Zalenskaya I, Hagver R, Azim A, and Gross R. 2005. Sophorolipids: Microbial glycolipids with anti-human immunodeficiency virus and sperm-immobilizing activities. Antimicrob. Agents Chemother. 49: 4093-4100.

Sharma SK, SaiRam M, Ilvazhagan G, Devendra K, Shivaji SS, Selvamurthy W. 1996. Mechanism of action of NIM-76: a novel vaginal contraceptive from neem oil. Contraception, 54: 373-378.

Staford MK, Ward H, Falangan A, Rosenstein IJ, Taylor-Robinson D, Smith JR, Waber J, Kitchen VS. 1998. Safety study of nonoxynol-9 as a vaginal microbicide: evidence of adverse effects. J Acquired Immune Defic. Syndr. Hum. Retrovirol., 17: 327-331.

Trussell J. 1994. Contraceptive efficacy of barrier contraceptives. In Barrier Contraceptives, Current Status and Future Prospects, Mauck CK, Cordero M, Gabelnick, HL, Spieler JM, Rivera R (eds). Wiley-Liss: New York; 17-52.

UNAIDS. 2004. The Joint United Nations Programs on HIV/AIDS (UNAIDS) and World Health Organisation AIDS epidemic update [online]. Available from www.unaids.org/ward2004/EPIupdate200 4_html_en/epi04_00_en htm.

Weitlauf HM. 1994. Biology of implantation. In The Physiology of Reproduction, Knobil E, Neil JD (eds). (Vol. 1, 2 ${ }^{\text {nd }}$ edn). Raven Press: New York; 391-440.

Zaneveld LJD. 1994. Vaginal contraception since 1984: chemical agents and barrier devices. In Contraceptive Research and Development 1984 to 1994. Van Look PFA, Perez-Palacios G (eds). Oxford University Press: Delhi; 69-90. 